\title{
Using SAGES OpenESSENCE for Mass Gathering Events
}

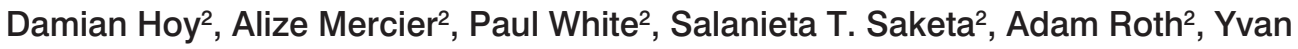 \\ Souares ${ }^{2}$, Christelle Lepers ${ }^{2}$, Richard Wojcik*1, Aaron Katz ${ }^{1}$, Timothy Campbell ${ }^{1}$, Shraddha \\ Patel $^{1}$, Brian Feighner ${ }^{1}$ and Sheri Lewis ${ }^{1}$
}

${ }^{1} \mathrm{JHU} / \mathrm{APL}$, Laurel, MD, USA; ${ }^{2}$ SPC Int, Suva, Fiji

\section{Objective}

Present how a surveillance tool such as SAGES was used for disease surveillance for mass gathering activities.

\section{Introduction}

The SAGES (Suite for Automated Global Electronic bioSurveillance) team at the John Hopkins University Applied Physics Laboratory was approached by the Public Health Division of the Research, Evidence and Information Programme of the Secretariat of the Pacific Community (SPC) to explore the feasibility of using the SAGES disease surveillance toolkit for two mass gathering events, the $8^{\text {th }}$ Micronesian Games held from 19-31 July in Pohnpei Federated States of Micronesia and the $3^{\text {rd }}$ International Conference on Small Island Developing States (SIDS) held from 1-4 September 2014 in Apia Samoa.

Mass gatherings are congregations of large numbers of people in a specific location(s) for a defined period of time such as major sporting events, concerts and festivals. A downside of these gatherings is the potential for communicable/infectious diseases to spread efficiently and rapidly. Infected individuals may subsequently return home and disseminate these infections in their local populations.

The Pacific syndromic surveillance system commenced in 2010. Enhanced syndromic surveillance is increasingly being used in mass gatherings. This involves inclusion of more syndromes and more regular reporting than routine syndromic surveillance. While enhanced syndromic surveillance is an important mechanism at mass gatherings, also, and somewhat opportunistically, mass gatherings can provide a unique chance to initiate or strengthen existing surveillance systems.

\section{Methods}

SAGES is a collection of modular, open source software tools designed to meet the challenges of electronic disease surveillance in resource-limited settings. SAGES uses mobile and web-based methods to collect structured data from SMS, Wi-Fi, and Internet connected devices. The primary data analysis tool, OpenESSENCE $(\mathrm{OE})$, provides a web-based interface with data analysis, visualization, and reporting. These tools may be used in concert with existing surveillance applications or be used en masse for an end-to-end biosurveillance capability.

SAGES OE can augment enhanced syndromic surveillance by providing a web-based data entry and analysis environment, which enables multiple data entry points, and more timely processing of data. Which, ultimately, enables the preparation of daily situation reports during and around the mass gathering.

For the Games, OE was hosted remotely because of a very short deployment and testing window. It also afforded the SAGES and SPC teams access to the web application to customize data entry forms and visualizations. For this event, the SPC explored the feasibility of wireless tablet data entry at the point of care event aide stations. However, due to connectivity coverage issues at several remote sites, paper forms were used instead for all locations. The forms were collected and the data entered daily into the OE web app at a central location.

Following the Games, a request to deploy OE for the SIDS Conference was made. The information collected was similar to the Games and required minimal changes to the entry forms to reflect the Conference's reportable conditions and point of care locations. However, for this event, the Samoan Ministry of Health decided to host an $\mathrm{OE}$ web site on their IT infrastructure.

\section{Results}

The SAGES OE tool was used for disease surveillance in two different mass gathering events. OE was adapted in less than a couple of weeks to fit the needs of the Games and the Conference. Visualizations used during the Games and for daily sitrap reports and can be found at the following URL http://www.spc.int/phs/PPHSN/ Surveillance/Mass_gathering.htm

Similar visualizations are planned for the Conference.

$\mathrm{OE}$ was generally very well received and provided a highly useful tool for assisting with detecting and containing the spread of communicable/infectious diseases. Sustainable improvements with syndromic surveillance systems are likely with longer lead (6 months) in trial periods.

\section{Conclusions}

The SAGES electronic disease surveillance tools are designed with an eye toward ease of installation and use, with an ultimate goal of creating a self-sustaining SAGES users community. We believe that tools such as SAGES tools can be rapidly deployed (although longer lead in times are preferable) to monitor mass gathering events as well as facilitate local and regional electronic disease surveillance and increased WHO IHR 2005 compliance.

\section{Keywords}

Mass gatherings; Disease Surveillance; Biosurveillance

\section{Acknowledgments}

Federated States of Micronesia, Pohnpei Department of Health Services Samoa Ministry of Health

\section{*Richard Wojcik}

E-mail: richard.wojcik@jhuapl.edu 